\title{
Properly Interpreting the Epidemiologic Evidence about the Health Effects of Industrial Wind Turbines on Nearby Residents
}

\author{
Carl V. Phillips, PhD \\ Populi Health Institute
}

July 19, 2011

*This is a preliminary draft of the following article in press:

\begin{abstract}
Carl V. Phillips, "Properly Interpreting the Epidemiologic Evidence about the Health Effects of Industrial Wind Turbines on Nearby Residents," Bulletin of Science, Technology, and Society, vol. 31, no. 4 (August 2011), pp. 303-315.
\end{abstract}

\begin{abstract}
There is overwhelming evidence that wind turbines cause serious health problems in nearby residents, usually stress-disorder type diseases, at a nontrivial rate. The bulk of the evidence takes the form of thousands of adverse event reports. There is also a small amount of systematically-gathered data. The adverse event reports provide compelling evidence of the seriousness of the problems and of causation in this case because of their volume, the ease of observing exposure and outcome incidence, and case-crossover data. Proponents of turbines have sought to deny these problems by making a collection of contradictory claims including that the evidence does not "count", the outcomes are not "real" diseases, the outcomes are the victims' own fault, and that acoustical models cannot explain why there are health problems so the problems must not exist. These claims appeared to have swayed many non-expert observers, though they are easily debunked. Moreover, though the failure of models to explain the observed problems does not deny the problems, it does mean that we do not know what, other than kilometers of distance, could sufficiently mitigate the effects. There has been no policy analysis that justifies imposing these effects on local residents. The attempts to deny the evidence cannot be seen as honest scientific disagreement, and represent either gross incompetence or intentional bias.
\end{abstract}




\section{Introduction}

There is overwhelming evidence that large electricity-generating wind turbines (hereafter: turbines) cause serious health problems in a nontrivial fraction of residents living near them. These turbines produce noise in the audible and non-audible ranges, as well as optical flickering, and many people living near them have reported a collection of health effects that appear to be manifestations of a chronic stress reaction or something similar. However, many commentators (dominated by those who stand to profit from national government subsidies for building wind turbines, particularly energy companies and local governments) have repeatedly claimed that there is no evidence of risk. This appears to be widely believed by those unfamiliar with the evidence but who believe that turbines are an eco-friendly energy source (a claim that is subject to debate) and think that anything "green" must be harmless to people.

While it is typical for industries and their supporters to downplay risks and argue that the benefits make the risks worthwhile, the wholesale denial of the evidence by both business and government in this case is reminiscent of such claims as "there is no evidence that smoking causes cancer" or "Iraq has weapons of mass destruction". However, unlike most industry denials or casus belli, where critical thinkers know to exercise some skepticism before accepting the claim, the denial of the evidence of turbines seems to have produced widespread credulity among those who would be expected to know better. This may be because the epidemiologic evidence is complicated and the attempts to deny it sound like the language of science. In response to that abuse of science, the goal of this paper is to empower interested observers to understand the nature and quality of the epidemiologic evidence and the weakness of the common arguments used in attempts to deny it. 
It is argued here that there is ample evidence that turbines cause a constellation of health problems, and attempts to deny this involve claims that are contrary to proper methods of scientific inference. Moreover, there is no basis for claiming that current regulations and recommendations are sufficient to avoid substantial risk, and those who claim otherwise do so without any basis. Indeed, ironically, what is often presented as evidence that there are no risks shows no such thing, but does demonstrate that most claims about what constitutes sufficient regulation cannot be scientifically. Moreover, the balance of the necessary ethical analysis seems never to have been performed. Given these observations and consideration of public policy ethics, it is difficult to see how most of the ongoing siting of new turbines can be justified.

\section{A brief review of the epidemiologic evidence}

For those not familiar with the term, epidemiology refers to the study of health outcomes and exposures in people for purposes of making assessments about population health. The critical feature is the studying of actual health outcomes in actual people, as opposed to other sciences (like toxicology or, most relevant in the present case, acoustics) which might help predict health outcomes but can be quite wrong about them. Those other sciences sometimes suggest possible effects that the epidemiology shows do not actually occur to a measurable degree and other times fail to predict the health effects that really do occur. Epidemiology is a quantitative science, though the quantification ("effect estimates") that is the preferred endpoint for most epidemiologic research is not always possible, as in the present case. Most, but not all epidemiology, focuses on whether a particular exposure (possible cause experienced by people) causes a disease outcome. While epidemiology often depends on observational evidence, 
sometimes experiments can be done; clinical trials are the most familiar, but a different kind of experiment has been done in the case of turbines.

There are many different types of evidence that contribute to epidemiologic knowledge. While the majority of formal epidemiologic studies use only three or four study designs, there are numerous other types of studies and sources of information. As with any science, when engaging in a directed inquiry to answer a particular question, one uses what information is available and purpose-builds further information-gathering, and often such information and study design differs from the most common study types. Indeed, in the present case some information is available from the common study types, but the vast majority comes from other sources, particularly adverse event reports (a particular type of what are known as case studies, sometimes denigrated as "anecdotes", that generally report on the rapid onset of a disease which appears to be related to a particular exposure) many of which involve case-crossover experiments. Both of these are useful and well accepted sources of epidemiologic information, and since they are intuitively recognized by both experts and lay-people seeking to assess whether an exposure is causing disease outcomes, people have collected this information for years (though it is not clear that anyone working in the area had identified the established terminology and it established history in the science value before I pointed them out last year).

\section{Large collection of evidence}

Most of the adverse event reports are self-published by those concerned about the health effects experienced by themselves or family members in the interest of contributing to public health knowledge on the subject. Most of these are yet to be organized, although efforts are underway. Others have been collected more systematically, such as the WindVOiCe collection 
from Ontario (Krogh et al. 2011), the scholarly book by Pierpont (2009), and in a paper by Harry (2007). Since several research groups and NGOs have collections that number in the threefigure range, it seems safe to conclude that the total number published or collected in some form is in the four-figure range, and it is quite conceivable that the total number of adverse event reports numbers in five figures.

Excerpts from three of these from my research group's collection appear in the Appendix to illustrate some of the points that follow. These three were self-published by the authors on the web and are fairly typical, though they were chosen because they were good examples, not because they are somehow perfectly representative of the collection. The reports have been abridged to remove information not presently relevant, and to reduce length.

In cases of emerging and unpredictable disease risk, adverse event reports are the cornerstone of public health research. Since it is obviously not possible to study every possible exposure-disease combination using more formalized study methods, just in case an association is stumbled upon, collecting reports of disease cases apparently attributable to a particular exposure is the critical first step. The most familiar examples of hazards revealed by adverse event reporting are infectious disease outbreaks or side effects from pharmaceuticals, but the case of turbines and health also fits the pattern. Pharmaceutical regulators rely heavily on clearinghouses they create for adverse event reporting about drug side effects (and often become actively concerned and even implement policy interventions based on tens of reports). The WindVOiCe report collection is an example of this same well-accepted kind of active-recruiting data collection system. 
As explained in the next few sections, useful self-reporting of adverse events is only possible for particular types of exposures and outcomes, but exposure to turbines and many of the reported health effects are just those types of exposures and outcomes.

\section{Reasons the adverse event reports are compelling}

Adverse event reports are under-appreciated as a source of evidence. The main reason for this seems to be overgeneralization from cases where they are indeed uninformative by those who do not understand what characteristics exposures and outcomes must have for them to be informative. It is always possible to find a single case study of an exposure-disease combination because even if there is no relationship between an exposure and a disease, it is statistically inevitable that someone will have both by coincidence. Thus, when political activists dig up a story about one such individual ("here is someone who had that exposure, and look what happened to him!"), we should be skeptical. This is especially true when the disease in question occurs frequently in the population and it is not possible to simply "see" the exposure that triggered it, like common cancers or heart disease. Many people get those diseases (and thus it is not difficult to find a few examples), the exposures that trigger them are invisible, and we cannot identify the onset to associate it with a proximate exposure. The challenge is greater still when the exposure itself is vague and difficult to precisely define, like "lived near the chemical factory". In such cases, it is nearly impossible to learn much from reports of adverse events, and indeed claims about a particular cause of one person's case of the disease can almost never be justified, and so more systematic studies are needed.

The reports about the effects of turbines are not such a case. The sheer volume of reports elevates the evidence beyond the few coincidental cases that can usually be found. The quantity 
further tells us that the effects go beyond a few rare individuals who are extremely susceptible. It is a legitimate limitation of adverse event reporting, no matter how voluminous, that it does not allow an estimate of what portion of the exposed population suffers health effects. There are undoubtedly similar effects among those who have not made the effort to publish the information, but we can only guess how common they are. It should be noted, however, that pharmaceutical regulators often make decisions based on exactly that guess.

Moreover, most reported health problems are similar across reports and are plausibly related to each other and the exposure. As illustrated by the examples in the appendix, there is a core list of symptoms - sleep disorders, headaches, mood disorders, inability to concentrate, tinnitus, vestibular (balance) problems - appearing in most reports. The commonly reported problems all exist at the border of the psychological and physical, and can all be caused by either of two very plausible effects of wind turbine exposure: stress reactions or vestibular disturbance. There are also a few reports of hypertension and other mortality-causing conditions, though since this is difficult for individuals to monitor themselves it would be unlikely to appear in most adverse event reports.

The Appendix examples also illustrate that some people attribute various other conditions they are experiencing to the turbines; this is not surprising, but the volume of reports lets us sort out rare coincidences (which can indeed generate misleading "anecdotal evidence" if a single story is treated as overly informative) from common patterns. We need not, and should not, simply accept the assertion of one individual or their clinicians about causation, assertions which appear in most of the adverse event reports. Rather, we focus on the consistent patterns of diseases that occur after exposure onset. (It is possible that mining the case reports more thoroughly will reveal apparent associations to diseases that were not previously believed to have 
been caused by turbines; such research is ongoing.) If people were complaining only about a collection of seemingly unrelated ailments, without the core overlap, it would suggest that they were just blaming the turbines for all their coincidental problems. But that is clearly not the pattern that emerges.

Most important, in contrast with exposures like invisible chemicals and diseases like cancer, individuals are capable of recognizing both the exposure and outcomes. Local residents are quite capable of observing that they are detecting noise or other effects. Moreover, people are capable of detecting their own insomnia, mood disorders, inability to concentrate, etc. Even more importantly, they are capable of detecting the incidence (i.e., onset) of these problems as well as when they cease, and while these problems are fairly prevalent in the population at any given time, their incidence is relatively uncommon and very often has a proximate cause.

For a relatively common condition, if we only had prevalence measures (i.e., how many people have the condition at any point in time), then in order to conclude that those living near turbines have a higher rate of the condition we would want to compare their rates to that of similar people not living near turbines. Similarly, if we were talking about cancer (where epidemiologists refer to the diagnosis as the incidence for convenience, but actually have no idea when the cancer initially began growing) we would want such a comparison. But for something that is very rare (e.g., not having a pattern of severe insomnia, which is not rare, but having that pattern start a particular week, which is) we can conclude the incidence rate is elevated without an explicit comparison. For example, many people have headaches at any given time, but if you have one that started at the time you suffered a trauma there is a good chance the trauma caused it because the probability of a headache starting at just that minute by coincidence is very low. Thus, people are quite capable of determining that incidence of these health problems occurs 
after exposure to moving wind turbines begins, unlike claims about what caused a particular cancer where such observations are not possible. Some supposed experts who have merely memorized a few simplistic rules of thumb from first-year epidemiology classes are unlikely to understand this, but the knowledge of that incidence and its timing is compelling evidence of causation even without a formal comparison group.

The above observations alone show that the adverse event reports are strong evidence for a causal relationship. The fact that many of the published adverse event reports include casecrossover observations and experiments push the evidence beyond a hint of plausible doubt.

A case-crossover study is one of the most compelling sources of epidemiologic data. It consists of observing whether someone's outcomes change as their exposure status changes. This is often not possible because the outcomes only happen a single time as a result of longterm exposure (e.g., cancer) or the exposure cannot be changed. But the observed effects of turbine exposure lend themselves perfectly to such studies because the exposure is transient and the effects, while not instantaneous in their manifestation or dissipation, are generally transient over a period of days or weeks at most. Thus, unlike a case of a lifelong exposure or nontransient disease, where we can only make one observation about disease and outcome per person, the effects of turbines allow multiple observations by the same person, including experimental interventions.

The case-crossover study design was first formally documented as a method for epidemiologic inquiry by Maclure (1991) though undoubtedly it was recognized as extremely useful for drawing conclusions about health effects from before the time our ancient ancestors achieved the rank of H.sapiens. A case-crossover study is the most natural form of scientific inquiry: "I ate that and my stomach hurt; I did not eat it again for a while and had no problem; I 
ate it again and my stomach hurt again; I think I there is a causal relationship here". This natural understanding of scientific inference is why such a large portion of the adverse event reports include crossover data. People observe, and report, that the exposure stops when the wind is not blowing or the subjects remove themselves from the area for a while, or especially when they relocate, and they observe whether the health problems abate. Some of these crossovers are observational (the change in exposure status was unplanned) but many are experimental (people intentionally avoid the exposure for a while to see if their health problems abate). Most of the reported crossover data confirms the causal inference that comes from the initial crossover from unexposed to exposed, the start of operations of the nearby turbines, which would be the only observation possible if it were impossible for exposure status to change again (as it is with such exposures as "ever smoked", "received a high dose of radiation", or "got older"). The examples in the Appendix include several of the common versions of crossover data, including complete relief upon relocating, the ability to sleep well when staying somewhere other than the subject's own home, and reactions to whether the wind was blowing through the turbines at any given moment.

An additional feature of the data in this case is revealed preference information about individuals' conviction regarding the causal relationship and intensity of costs inflicted upon them. Many people report expending substantial resources - retrofitting their houses to reduce noise, selling their properties at a loss, or even abandoning their homes without being able to sell them - in order to try to reduce the health impacts. (The Appendix includes examples of such revealed preference.) Thus, rather than just claiming they were confident about the causal relationship (perhaps thanks to personal case-crossover experiments) and describing the intensity of their suffering, they "put their money where their mouth is" and endure great expenditure, 
demonstrating great confidence in their assessment and that the magnitude of the suffering warrants such expenditure. Similar revealed preference can be found in the inability of owners of property near turbines to be able to sell it at a price comparable to other homes or land that is not near turbines. It is sometimes claimed that few people believe there are harmful effects or that they would experience them if they lived near turbines, but property values and sales collapse only if almost everyone is uninterested in living there. If merely a few people believed the claims that there were no problems, and were willing to intentionally relocate to live near turbines, then they would take advantage of the bargains and move in; alternatively, speculators - perhaps the energy companies or local turbine proponents - would snap up the bargains. This is apparently not happening, suggesting that no substantial number of people, even those making the claims, genuinely believe that the turbines are harmless.

In sum, the present situation lends itself perfectly to having useful adverse event data, in terms of exposure and outcome that are easily identified, incidence times that are easily identified, the possibility of case-crossover data, and the possibility of revealed preference. The empirical reality is that we have an enormous volume of data, the outcomes reported are plausibly related, many people have performed case-crossover experiments that support the conclusions, and there is indeed revealed preference data.

This still leaves the inherent limitations of this type of non-systematically gathered data: Because the data does not have known sampling properties from a well-defined population, it does not itself tell us how many others might have reported adverse events had they experienced them, but did not. This information is the denominator that would be required to calculate the portion of all exposed people who experienced the adverse events. We have some information that lends itself to estimating that figure, from a handful of systematic studies and using 
estimates from where there was a focused effort to collect all local adverse event reports, but not as much as we would like. The data we have also offers limited opportunity to estimate how much the risk changes with characteristics (in particular, that means we do not know how far away turbines need to be from residences to reduce the risk below some particular level).

Nevertheless, in terms of demonstrating that there is a substantial risk of serious health problems, the adverse event reports are more compelling than any small number of systematic studies could be. Moreover, the sheer volume of data makes it possible to mine it for some information that is normally only available from systematic studies, like the effectiveness of crossovers at eliminating the health problems and the dose (distance) response; such mining is underway.

\section{Systematic epidemiologic evidence}

While not providing as much information as the adverse event reports, the few systematic studies (with data gathered from a defined population, allowing calculation of outcome prevalence), offer the reassurance that different sources of information support the same conclusions.

There is a small collection of systematic studies from Europe by Pedersen and colleagues (2004, 2007, 2009, 2010). These studies suggest that some substantial portion of exposed individuals experience harms, some of which constitute health problems by any modern definitions of health. The studies have various limitations, but they provide a quantification of a nontrivial number of cases. Phipps (2007) also tends to support the claim. Nissenbaum et al. (2011: personal communication) was the most effectively purpose-built study to date. It surveyed residents living near turbines about most of the above-mentioned health conditions and 
compared them to similar people living further away. The results (unpublished at the time of this writing) appear to support most of the widely-stated hypotheses about the health effects of nearby turbines. Importantly, there do not appear to be any systematic studies whose results suggest conclusions contrary to those we draw from the adverse event reports. In particular, there is no evidence to support the claim that the relevant health problems are similar in unexposed and exposed populations.

\section{Attempts to deny the epidemiologic evidence, and their fundamental flaws}

Because of the revenue that is at stake from government subsidies to wind power producers (including to land owners and local governments), there are wealthy organized interests who would prefer everyone doubt that the observed health problems exist. There also appears to be some "not invented here syndrome", with some acousticians and clinicians resenting the fact that they cannot explain the observed health problems, and thus seeking to deny the epidemiology. Whatever the motivation, there has been a pattern of anti-scientific claims aimed at denying the evidence. While there is individual variation, a pattern has emerged within the denial tactics (and that word choice is intentional: it is difficult to see these as anything other than directed efforts to secure a particular goal). The following divides these into three categories and explains why each of the common arguments is groundless. While this does not cover every single argument anyone makes, it is comprehensive in the sense that I am aware of no common or potentially-credible argument against the validity of the epidemiologic evidence that is not addressed here.

\section{Pretending that there is no evidence of health effects}


Many pro-turbine reports sponsored by industry or government simply ignore all of the adverse event reports. Any reader limiting himself to those supposed summaries of the evidence would not even be aware of the majority of the evidence. Obviously this is blatantly anti-scientific conduct. It is perfectly legitimate to argue that the ostensible evidence against your claim is uninformative or wrong, but anyone pretending it does not exist is attempting to mislead their readers and is presumably not confident their claims can stand up to the evidence.

When turbine proponents acknowledge the evidence and seek to deny its usefulness, they are more honest, but no more convincing. Many such denials translate roughly into, "we do not recognize the informative value of adverse event reporting and case-crossover studies, and therefore we are going to declare they are not informative." In practice this differs little from simply pretending there is no information - no one ever admits, "there are thousands of case studies that report crossover data that correspond to the causal claim", before going on to argue that we should not believe them. But it bears separate mention because it is common, particularly among the epidemiologists from consulting firms that industry likes to hire. Their claims are not merely that some types of evidence are more useful than others when answering a particular question (which is true) or that there is some rigid epistemic hierarchy wherein one type of study always trumps another (which is false, but is commonly believed by non-experts who know a bit about the subject), but sometimes that there are only two types of epidemiologic studies.

These authors' limited understanding of epidemiologic methods may stem from the fact that most of their work is defending against claims of cancer and other diseases that have the characteristics described above, where it is difficult (but not impossible) to learn much from anything other than one of those two types of studies. What they do not seem to understand is 
that exposures and diseases are often not epistemically similar to chemical exposures and cancers. The evidence that is most useful depends on the question being asked. (For more on these points and others in this section about how to interpret epidemiologic evidence, see Phillips 2011.)

So, to pick a recent example, if many people gathered in a North African city started sending out messages that government forces are shooting into the crowd, it is very good evidence that shooting is happening. Yes, we could do a controlled study to find out if the number of gunshot wounds treated at hospitals was higher that day than the same day the year before. But it is possible that such a study could miss the effect - it could sample from the wrong hospitals, or the government could act to suppress the information, or the study could suffer from any number of problems. It is true that some systematic survey, rather than individual reports, would be necessary to quantify the toll. But the event reports coming from eyewitnesses would be the most definitive source that the event was occurring, and if they were provided in enough detail by enough people they would be systematic enough to be the best way to estimate the toll. To claim that we know nothing about those events until we have multiple systematic studies (what some turbine industry proponents have argued) requires a willful suppression of normal human reasoning. This example is not meant to be a cartoon; it is actually a reasonable analogy. In terms of witnesses' ability to detect the exposure and outcome and the potential limitations of more systematic studies, turbines are much more similar to gunfire than they are to the effects we normally attribute to noxious facilities.

The other common method for trying to claim that the adverse event reports are not informative (again, typically coupled with trying to imply there are only handful of them, failing to acknowledge the quantity) is to note that they are not "peer reviewed". It seems likely that 
most people repeating this claim do not even understand what peer review does; if this is not the case then they are pretending not to understand. At its best, peer review by health science journals provides a cursory review to make sure that a study follows some basic guidelines, and occasionally (very rarely) corrects an important error. Reviewers rarely comment on the quality of analysis methods, let alone the data being analyzed, because they do not even provided with the statistical programs or data. Reviewers see nothing more than what eventually appears in the journal, which almost never allows the reader to know critical features of the study design, let alone assess whether they were carried out adequately. And that is the best case scenario; more typically peer review in the health science does more to censor politically incorrect evidence and discourage innovation than it does to improve what is published. It is easy to get an absolutely terrible study published, so long as the authors jump through particular hoops, stick to simple methods, and do not reach a conclusion that is controversial (the conclusion need not follow from the data, however). It is quite difficult to publish a high-quality innovative study that engages if more complex analysis or demonstrates something new - e.g., the present analysis or WindVOiCe. Moreover, peer review has drifted from being a minimal but useful gatekeeper to primarily being a method for university employees to keep score for their annual reviews.

That is all it is, and treating it as more fetishizes it, in both of the technical senses of the word: It is treated as some kind of magical process, and it has become a stand-in for the phenomenon (good and accurate research whose essential elements have been assessed and improved by multiple experts) that it is supposed to represent. While it may be necessary for casual readers with absolutely no expertise in a field to defer to rules of thumb like "only look at the peer reviewed literature and 'expert' reports" to avoid falling victim to the worst quackery, that merely means that it provides some filtering, not that the rule is a useful guide for serious 
scientific inquiry. A debating society or a grade school term paper needs rules to structure the artificial exercise, and so can just as easily choose "peer reviewed references only" as any other rule. There are also arguments to be made for (and against) the use such rules of evidence in liability trials and other formal processes that need some rules of conduct. But there is no justification for legalistic rules of evidence when engaging in scientific inquiry and its extension, science-informed policy making.

Moreover, even though value may be added from peer review of the best kind (which usually takes the form of circulating a paper among colleagues, not relying on the triage system of the journals to add much value), no peer review can vouch for the accuracy of data without actually reviewing the data collection method. This means that adverse event reports, which consist of someone reporting their data as best they can (we can ignore the authors' own analyses and conclusions and focus just on the events data), would not be improved by peer review. That is not to say that the adverse event reports will never be represented in the peer reviewed literature; they will almost certainly become the data for analyses in journal articles, but will not be rendered any more accurate as a result of that.

Indeed, eventually most of the useful information on a topic is referenced somewhere in journal articles, and at that point relying only on those articles alone does not cost us too much information, but we are currently far from that point for this topic. For supposed experts, acting as consultants or otherwise writing "expert" reports, the fetishization of peer reviewed literature is basically a concession by the authors that they are not really experts in either the relevant scientific methods, the subject matter, or both. Actual experts are the ones who can look at something and assess its usefulness; after all, being an expert means being capable of performing the peer review rather than relying on someone else's assertions. 
Moreover, until there is a sufficient body of literature, the adverse event reports will continue to be more compelling than the formal studies. Anyone who is familiar with epidemiology knows that it is easy to design a study that, as reported in the cursory description of methods that peer reviewers ever see, reads like it is solid and would detect the phenomenon of interest if it exists, but is actually almost guaranteed to find nothing. Consider how easy it would be to conduct the study, "I searched the apartment for my keys but did not find them", in a way that would likely fail even if the keys were in your apartment (e.g., search for only five seconds; search the neighbor's apartment instead of your own; keep your eyes closed; put the keys in your back pocket before you start). Designing a study to fail to find a health effect from turbines would be equally easy, and it is actually a bit surprising that the industry has not done this already.

It is also quite possible to design a study that is likely to "detect" a phenomenon that does not exist. This is a bit trickier than intentionally finding nothing, and sometimes requires detectable subterfuge like defining-down the phenomenon studied to something common and unimportant but then reporting the result as if it were dramatic (e.g., asking "have you ever noticed turbine noise while trying to fall asleep" and then reporting "our results show turbines cause sleep disorders"). Setting aside the details and the direction of likely bias, the general lesson is that in an advocacy situation, opaquely complex studies of the type published in health science journals can easily be gamed by a researcher without even violating the rather weak norms in the field, while gaming thousands of adverse event reports would require massive fraud.

Finally, it can be argued that those who seek to deny the evidence are making an implicit promise to believe the evidence of health effects as soon as it appears in peer reviewed journals, 
and they should be reminded of that implicit promise in the future. Most people reading this paper will do so after it has the imprimatur of "peer reviewed publication". That means that the adverse event reports contained in the Appendix are peer-reviewed journal publications. Does that make them any more valid than when originally published by the author? Of course not. The reviewers had no way to assess the accuracy of the report, just as reviewers of health science papers can never vouch for the accuracy of the data that underlies an analysis. But those who protest that lack of peer review is the reason for not believing much of the evidence are now obliged to accept the report in the appendix as fact.

\section{Trying to claim that theory and historical guesses outweigh the evidence}

If no one could figure out any way that turbines could possibly affect people's health then we would have reason to seriously question whether the epidemiology was correct. But we know that noise and light effects from turbines impact people's senses and otherwise affect their bodies, and so there is a plausible causal pathway from the turbines to diseases. Furthermore, we know that some of the impacts create distressing awareness which causes stress reactions, which might or might not explain many of the observed health problems, but it plausibly could explain them. There are also more complex theories about pathways that are the subject of debate by those who are expert in the biophysics.

Thus, it is not yet clear which of the hypothesized pathways play a significant role in health effects. But it is clear, given the list of plausible candidate pathways, that there is no legitimate basis for claiming it is impossible for turbines to cause health effects. Yet that is exactly what some commentators have claimed. Generally this takes the form of someone proposing one pathway by which noise might cause health problems, probably the one he has 
studied, and offering a single model for assessing whether the outcome is likely to occur. When his model cannot explain the problems, he concludes that there are no problems.

The flaw in this reasoning should be obvious, though it is remarkable how convincing some people apparently find it. Sciences like toxicology and, in this case, acoustics can help predict whether an exposure might cause a health problem before we have actually observed whether it does so. But the epidemiology trumps the predictive evidence. Acoustics and other science still have an important role to play in helping figure out why the turbines are causing health effects, but their role as predictive sciences is over: we now have epidemiologic results which the other sciences apparently failed to predict. Continuing to rely on predictions about whether the exposure might cause disease once we have seen that it does cause it is like trying to figure out whether it is raining right now by looking at last week's weather forecast for today.

A particular observation takes the form of claims like, "there are health effects associated with living near a turbine, but the physical sciences show there are no direct effects." These assertions about "no direct effects" are presented as if the phrase has some scientific meaning, and thus the reader should be impressed. But anyone with expertise in studying causation will realize that the statement is vacuous because there is no such construct as "direct effect". It is always possible to insert an intermediate step in between two points in a causal pathway, or remove all the intermediates from consideration, and thus it means nothing to call an effect either direct or indirect. Moreover, though this is generally a component of strained arguments that are attempting to deny the evidence, it should be recognized that those making this claim are saying "the physical effects caused by the turbines impact people's bodies, but it is only when some biological or psychological process is caused by that impact that the diseases we observed are then caused". That admission that the turbines are causing health problems is certainly an 
accurate statement, just as it is accurate to say that cigarettes do not "directly" cause lung cancer because the cancer only happens when their impact on the body, interacting with other factors, triggers a complicated biological process that eventually causes a tumor.

There is one key lesson we can take from the inability of the physical modelers to agree about why the health outcomes occur, and the fact that there are steps in the pathway that we cannot yet be sure about: It is currently impossible to know how to change the exposure to mitigate the health effects with much confidence, other than by separating people and turbines by a great distance. The existing epidemiology does not answer this question either. Yet some proponents of turbines argue that the regulations that have been created in this state of ignorance must be sufficient to protect people. It should be obvious how absurd this claim is. Those regulations are a hodgepodge of different rules that are generally based on old recommendations that predate the recognition of the health effects and which, to the extent they were based on any science at all, were based on the science that incorrectly predicted that there would be no problem. Even if one believes that the regulations were as well thought-out as they could have been, the epidemiology shows that people suffer health effects when the regulations are adhered to.

A variation on this theme is to claim that the health problems exist only when current regulations are not met, or even when the original regulations under which the turbines are operating are violated. It is certainly reasonable to condemn operators and regulators who allow the violation of the regulations, a fairly common occurrence according to the adverse event reports. However, since those making the argument that current regulations should be adhered to do not seem to be offering such condemnation or conceding that turbines do sometimes cause health problems, it is difficult to accept their implicit assertions that adherence to current 
regulations is genuinely the difference between causing health problems and not doing so. But the argument that some set of more stringent regulations is sufficient to eliminate the health problems (or that it will be obeyed) is no better supported by science than the claims that previous regulations were sufficient. Moreover, there is no shortage of aphorisms about how unwise it is to believe someone who is telling you "we were wrong, or perhaps even lied to you, about the previous regulations being sufficient, but trust us when we assert, in exactly the same way, that everything will be fine with new regulations." This is especially true when they still do not even admit that the previous regulations were insufficient.

In any case, regulatory choices and guidelines are decisions that, at best, are sciencebased, but offer no scientific information in themselves. It should be clear that believing last week's government decree about what the weather should be today is even less useful for figuring out whether it is raining right now than would be looking at last week's forecast. Unless, that is, we are in an Orwellian world where the language is redefined to make government decrees always correct. Some of the tactics for denying the evidence of health effects actually seem quite similar to that.

\section{Claiming that the observed health effects are not really health effects}

Failure to understand how to draw scientific conclusions and myopia about a single method for modeling physical health effects are problematic, obviously. But they are not so clearly reprehensible, from an ethical standpoint, as telling people that their suffering does not really "count" for some technical reason.

A common claim is that the health outcomes do not constitute a "disease" because there is no officially recognized labeled disease along the lines of "wind turbine syndrome". But even 
if we set aside that the individual diseases people are suffering, like chronic stress and sleep disorders, are often well-defined (they are just not defined in terms of a specific cause), and the fact that the term "disease" is quite broad in practice, this would still only be a semantic point. There is no epistemic significance to the health outcomes in question having or not having a label.

Sometimes the denial takes the form of saying that people are just suffering "symptoms" but not a "disease", which is nonsense since in this context the word "symptoms" is shorthand for "symptoms of a disease" and, moreover, can roughly be defined as "those manifestations of a disease that are (in addition to mortality) what people actually care about". Another semantic game says that what people are suffering is not "disease" but "annoyance". The jargon used in the noise and health literature refers to effects of noise that are apparently psychologically mediated (i.e., most everything other than hearing damage) as "annoyance". The rhetorical game is to try to confuse readers into thinking this has the natural language meaning of "mere annoyance". However, the jargon meaning of annoyance includes everything from "mere annoyance" up through a life-ruining source of severe distress. Moreover, even "mere" annoyance can itself have severe health consequences when someone is forced to exist in a chronic state of annoyance.

Another somewhat common pseudo-argument is that we should dismiss the health effects because they are all "subjective". This is not actually true since, for example, insomnia can be objectively measured by looking at a clock and even long-term stress itself can be measured via hormone levels. The authors seem to be confusing "subjective" with "psychologically mediated", which most of the observed effects might well be (though there are hypotheses about non-psychological pathways). 
But being subjective or psychologically mediated does not mean these effects are minor or less real. Indeed, there is a case to be made that such diseases, which include everything from transient headaches to chronic pain and depression, account for the majority of the total burden of disease in our society.

A recent addition to the rhetoric that the effects are of no consequence are assertions that effectively assert, "the problems are caused by the victims' own psychology, not the turbines." It is claimed that perhaps people fear the turbines, as people are known to irrationally fear toxic chemicals, and it is fear, not the noise or light, causing all the problems. This is a variation on the theme that was popular a year ago (though it can still be found) where it was claimed, based on one study that found a correlation between people's attitudes toward the turbines and their reported health effects, that attitudes were causing the problems. These claims sound more scientific than some of the other rhetoric, but are easily shown to be wrong.

First, the assertions themselves are rather weak. The fact that there is a relationship between suffering health effects from an exposure and disliking the exposure hardly comes as a surprise. If those who were trying to deny the evidence had read the adverse event reports, they could have found much more compelling evidence supporting this point (the feeling is apparent even in the Appendix examples despite the abridgements focusing on outcomes rather than judgments); however, in most cases it is clear that the people's dislike of the turbines was a result of their physical effects, not vice versa.

We do know that people have irrational fears of invisible hazards like radiation or "toxins", but it is difficult to imagine why someone would find a fairly simple object and exposure (metal, tower, fan blade, motor/generator mechanisms, wind, noise, flashing lights) to invoke the irrational fears that often result from invisible spooky exposures. If someone were to 
try to make a plausible claim along these lines, a better case could be made that that sensitization to the serious effects of turbines is psychologically contagious; that is, once some people start to suffer serious health problems, those around them are more likely to suffer distress reactions that lead to sersious health problems. This is still a speculative hypothesis, though unlike the purely speculative claims about irrational fears, one that is supported by some evidence (as illustrated by one of the examples in the Appendix, serious health problems that are attributable to turbine exposure tend to run in families, which might be due to their similar genetics or exposures, but might be a contagion effect).

But whether or not contagion or fear occurs to some extent does not excuse the turbines. This is not a case, like some commentators have tried to portray, that is similar to many people working in a building getting sick, apparently due to mass hysteria since no contamination or infectious agents are found. Unlike a single building, people with no knowledge of or worry about the health effects from other turbines, and who have never known anyone who experienced those problems, have discovered they are having health problems when turbines were built near their homes. Moreover, everyone suffering health problems does detect and usually complains about the sensory effects from the turbines, so it is clear that their entire experience is not imaginary. It is, of course, possible that some personal characteristic sensitizes them to be more bothered by the sensory effects, increasing any psychologically-mediated effects. But it is inevitable that some personal characteristics will be causal cofactors (factors that, along with the turbine, are part of the necessary constellation of causes for there to be a disease effect). This is true for every exposure-disease combination: Some exposed people get the disease and some do not, and sometimes we can identify other differences between the two groups. None of this changes the fact that the turbines are causing disease, and are the one of the many causes that we 
refer to as "the" cause in a legal or ethical sense. Compare: If someone is killed in a robbery, the cause of death was the assailant, but the death was also caused by the victim being at the wrong place at the wrong time, and perhaps because those around him were not skilled medics and that he was frail. However, the murderer's blameworthiness is not diminished by (inevitable) existence of causal cofactors.

Some commentators who are unfamiliar with causal analysis in epidemiology seem to believe there is something unusual about personal characteristics being correlated with the outcome. They then compound their error by declaring that if there is something psychologically different about the people who suffer disease, then we can "fix" them with counseling. This has been presented as being a reason why we should not worry about the observed health effects, even though the usefulness of counseling is a purely speculative hypothesis (there is no evidence it has ever been successful in these cases), and one that is built on speculation about there being a "treatable" causal cofactor that is a necessary component of the causal pathways. To return to the lung cancer analogy, someday we will invent a drug that keeps the injured lung cells from turning into cancer, which would mean that smoking would no longer cause lung cancer among people who took the drug, but that is obviously not an argument that smoking is not really causing such harm now; indeed, we would probably still consider the propensity of smoking to trigger lung cancer as consequential even if the hypothetical drug were available.

Moreover, the fact that we have no reason to believe we can actually counsel away the suffering caused by turbines is only part of the problem with such claims. Even if the counseling were useful at reducing the health effects, causing people to need counseling (a substantial cost in their lives even if it is completely successful) can hardly be considered a harmless and ethically inconsequential act. It has complicated ethical implications that evoke the Orwell's 
teaching someone to love Big Brother, and at a more prosaic level it would not eliminate suffering from the moment of first exposure. We have counseling methods that can reduce the long-term damage that someone suffers from being a victim of sexual assault, after all, but its existence does not change the fact that the assault does damage, let alone does it cause us to decide to allow the assaults because the damage can be undone.

\section{Any claim that might stick}

It is worth pointing out the dishonesty inherent in presenting many of these claims together, as is often done. It is a legitimate tactic for a criminal defense attorney to argue that the prosecution has failed to show that his client was at the scene of the crime, and that the evidence showed that if he was there it was after the crime was committed, and also that he was just along for the ride and did not know anything about his friend's plan to commit a crime. However, this is not a legitimate tactic in scientific analysis. Proponents of wind turbines have claimed, often simultaneously, that the physical models show there is no possible problem, that there is no problem if some particular rule is obeyed, that there is no evidence of health effects, that the reported evidence of health effects does not count because it in the wrong format, that there is evidence of effects but they are not real diseases, and that the diseases are really the victims' own fault. They also sometimes argue that the benefits outweigh the costs, the point taken up in the final section below.

We can perhaps excuse lawyers who work for the industry for making this contradictory mélange of arguments, assuming that we think it is acceptable for industry to act as selfishly as a criminal defendant is expected to act, and that it is up to others to make the opposing case. But there is no such defense available to consulting scientists who are supposedly writing reports as 
independent experts, or to government officials. They should be conducting the best possible scientific analysis. If one of them really believes that, for example, we should not worry about the health effects because people can and should be counseled to get over them, then they are still obliged to recognize the enormous number of adverse event reports and point out that acoustical theories predicting no effects are apparently wrong, before then going on to argue that the effects are something that is easily fixed. Similarly, someone who genuinely believes that there is not evidence that people are suffering cannot also argue that this non-existent suffering has characteristics that make it less important. Some arguments can legitimately be made in combination, of course. But even honest activists, let alone honest scientists, do not use the approach of throwing every claim that could possibly be made in support of their position against the wall to see what sticks.

\section{Ethical policy decision-making}

Public policies often impose substantial costs on people. We generally try to prevent a large share of the burden from falling on a small number of identifiable involuntary victims (e.g., people living near the site of a new noxious facility), but sometimes this is not possible.

Generally in such cases it is considered ethically mandatory to compensate the victims. But even setting aside tricky questions of just compensation, there is the simple principle that the total social benefits should outweigh the costs, which include the health costs. There is currently no evidence to support the claim that this is true for the installation of new industrial wind turbines in populated areas.

It is beyond the present scope to even rough out such an analysis, but it is possible to provide the steps that are necessary and point out how nothing remotely sufficient exists. First 
the net costs and benefits from a purely industrial standpoint (the resource cost to install, maintain, and decommission the turbines and transmission lines compared to the value of the electricity generated) need to be calculated; this is presumably negative since the industry depends on subsidies of various kinds. A common claim is that this negative value is more than outweighed by the net benefits of pollution reduction, though there is substantial debate about this point. Before there is any possible justification for inflicting involuntary health risks on people, whatever their magnitude, it is incumbent upon the industry to present a convincing analysis that shows there are substantial net benefits when all benefits and costs - apart from those imposed on local residents - are considered. Only such net benefits could justify impose the local costs.

It does not appear that this has ever been done, but for purposes of the exercise assume it has and the result is indeed positive. Then the health and other local impacts need to be quantified and compared to that benefit. While there can be no perfect quantification of such effects, estimates are possible employing well-used straightforward methods. The first step is to admit that there is a problem, which might be made easier by dropping any disagreement about whether the suffering constitutes health effects, since all costs to the local community, health and otherwise, should be identified and quantified. If there are indeed methods for mitigating the damage, and if particular regulatory standards can substantially reduce it, then this should be demonstrated and then implemented to lower the costs. These costs, lowered as much as possible, should then be compared to the other benefits

Many of us have guesses about how this comparison will come out, but the main issue right now is that it has never been done. No one has even taken a rough cut at the numbers, and so there is simply no basis for claiming that the benefits justify the costs. Indeed, this may 
explain why many proponents of turbines insist on making the extreme and obviously incorrect claim that there are no health effects at all. Once some numbers are estimated, we can begin to discuss whether the tradeoffs are justified, how to offer justice, and other policy questions. In the meantime, it makes no sense to take expensive largely irreversible actions, rather than exercising some easily reversed prudent delay until we better understand the situation.

\section{Conclusions}

It is always possible that further research will reveal that, under certain circumstances, turbines can be sited near people's homes with minimal health risk. Such is always possible for any exposure, given the nature of science (open to additional information) and changing technology. But our current knowledge indicates that there are substantial health risks from the existing exposure, and we do not know how to reduce those risks other than by keeping turbines several kilometers away from homes.

Similarly, it is quite possible a public policy case could be made for the claim that the costs are justified by the benefits. But the key is that the case must be made, including a quantification of the impacts on local residents, which has not been done. Those who pretend that there are no serious impacts on local residents cannot contribute any useful analysis. Moreover, it seems unlikely that it will ever be considered ethically acceptable to force susceptible individuals to suffer serious health problems, to say nothing of the non-health complaints and effects on communities, without much greater and more reliable compensation than has been offered to date.

Dismissal of health effects cannot be seen as honest disagreements about the weight of the evidence. Honest disagreements about scientific points are always possible. But when 
proponents of one side of the argument consistently try to deny the very existence of contrary evidence, make contradictory claims, appeal to nonsensical and non-existent rules, treat mistaken predictions as if they were evidence of actual outcomes, play semantic games to denigrate the reported outcomes, and blame the victims, then they are not being honest, scientific, or moral. They are preventing the creation of optimal public policy and damaging the credibility of science as a tool for informing policy. Moreover, since their lack of plausible arguments suggests there are no defensible arguments to be made on that side of the issue, their persistence in making implausible arguments is directly responsible for hurting significant numbers of people.

Acknowledgment: The author thanks Catherine M. Nissen for research assistance. 


\section{References}

Krogh C et al. Wind Vigilance for Ontario Communities; A Self-reporting Survey: Adverse Health Effects, Industrial Wind Turbines (IWT) and the Need for Vigilance Monitoring. 2011. Available at windvigilance.com.

Harry, A. (2007). Wind turbines, noise, and health. February 2007. http://www.windwatch.org/documents/wp-content/uploads/wtnoise_health_2007_a_harry.pdf

Maclure M. The Case-Crossover Design: A Method for Studying Transient Effects on the Risk of Acute Events. American Journal of Epidemiology 133(2):144-153, 1991.

Michael A. Nissenbaum MA, Aramini J, Hanning C. Personal communication based on a draft of the currently confidential “Adverse Health Effects Related to Industrial Wind Turbines (IWTs) - a Retrospective, Cross- Sectional Epidemiological Study”. 2011.

Pedersen E, Waye KP. Perception and annoyance due to wind turbine noise; a dose-response relationship. Journal of the Acoustical Society of America. 116(6):3460-3470, 2004.

Pedersen E, van den Berg F, Bakker R, Bouma J. Response to noise from modern wind farms in The Netherlands. J Acoust Soc Am, 126(2):634-43, 2009.

Pedersen E, Persson WK. Wind turbine noise, annoyance and self-reported health and well-being in different living environments. Occup Environ Med, 
64(7):480-6, 2007.

Pedersen E, van den Berg F, Bakker R, Bouma J. Can road traffic mask sound from wind turbines? Response to wind turbine sound at different levels of road traffic sound. Energy Policy 38(5):2520-2527, 2010.

Pierpont N Wind turbine syndrome: a report on a natural experiment. K-Selected Books. 2009. 


\section{Appendix: Excerpts fromt three adverse event reports}

Case 1, first person report by male, 2007

The first 2 turbines were operating in may 2004. One was $4000 \mathrm{ft}$ from our home, the second 4700ft. A 120 day trial period was required to monitor their productivity. We could hear them well and ..., my wife was experiencing ringing in her ears. Visitors would comment that the one $4000 \mathrm{ft}$ away seemed really close. Some neighbors were complaining they were bothered by them at night.

February, 2005 the windfarm was fully operational, 17 wind turbines. The windmill $4000 \mathrm{ft}$ away seemed far off compared to the one $1000 \mathrm{ft}$ from our home. They are loud. They've been compared to jet engines, a plane that will not take off. There is no gentle swoosh, it is a whoosh noise. They grind, they bang, they creak. The noise is like surround sound, it's omnidirectional. It feels like there's this evil thing hovering above you and it follows you everywhere, it will not leave you alone. This noise will not allow you to have your own thoughts, the body cannot adapt, it's a violation of your body. It is a noise that the human body cannot adapt to even after more than a year of exposure. As time progresses the noise becomes even more unbearable. 
Our 5 year old son ... was afraid and unable to sleep in his own bed for more than one year. He would get in our bed or in his brother's bed. We would put him to bed at 8:30 and many nights at 11:00 he would still be awake. Finally he would fall asleep wrapped up in the blankets in the fetal position with his head covered and with a fan at his head. we had to create more noise to mitigate the windfarm noise. The body can adapt to the fan noise. In the morning he would get up tired and cranky. In september 2005 he started school and he was not getting enough rest. He began getting more and more aggressive with his friends. He was very defiant We knew he was suffering terribly. He's had throat infections and often had a fever and not feeling well.

We abandoned our home February 21st, 2006.

Since the move [aforementioned son] has been doing much better. He sleeps in his own bed every night. He sleeps partially covered with his arms and legs spread everywhere. It was only ten days after the move while he was having his back rubbed in bed he said "it's nice to be able to go to bed and sleep". He is much less defiant. He has become the kind gentle little boy he was before the windfarm nearly destroyed his life.

Knowing what we know now we should have moved a year before.

Our 9 year old son ... was sharing a room with [5-year-old]. He was also very sleep deprived. He would get up in the morning very tired. We would send him to school tired. He was tired and 
unable to concentrate and his school work suffered. He was also unable to concentrate on his homework. He began to withdraw within himself. He also began getting aggressive. [He] seemed to be always angry. His teacher asked us what was the problem with [him] because his change in behavior was something she would never have expected from him. [His] ear drum burst while we were there in 2005. He's had many throat infections and many headaches. He has developed allergies. He's the only one of 6 children that has allergies.

Since the move [he] has improved so much in his school work and his behavior and participation in class that the teacher says she cannot believe that he's the same child. He has not been aggressive with his friends. He's so kind and caring for everyone. His headaches are less frequent and less severe.

Our 13 year old daughter ... had dramatic behavioral changes. She became withdrawn and was spending too much time alone in her room. She dropped her friends and lost interest in school work. She was also angry. She dropped all sports (basketball, volleyball. soccer, badminton). [She] always had headaches. She became very defiant.

Since the move [she] is doing better in school. Her behavior is steadily improving. Her health is improving and she is socializing. She is feeling better about herself.

[...reports on other family members omitted for length...] 
As for myself I always felt a sensation in my chest which was very discomforting. On extremely rare occasions when the windfarm was off I could sense they were off without seeing them. The noise was just a relentless attack on our bodies. Every time the blades passed the towers I could feel it within my body. I was unable to concentrate well enough to read in my bed.

Since the move I don't have that sensation in my chest but it returns when I spend a few hours at our house.

These physical and psychological effects develop gradually and sometimes it seems silly to associate them with a windfarm until you learn that others experience the same thing under similar conditions.

If we would have had absolutely nowhere to go, if we would have been forced to stay in our home, I hate to think what kind of physical and mental state we would be in now.

During the months that the sun is low in the sky we get a flickering in the morning and late afternoon as the sun passes behind the turbines. This induces headaches quickly to those who are more susceptible to them. When the full moon rises and passes behind the turbines the flickering is intense.

We are devastated, we are broken because we have lost the home we built with our own hands and we have lost the land which has been in the family for generations. 
Our house is now unsellable. There is nobody in the community that wants to live there because of the windfarm. Nearly everybody supports us privately but they are afraid to speak out publicly.

We are a community of 2000 people and I did a survey of 216 people and $96 \%$ said the windfarm was too close to [their] house. Also $89 \%$ said the windfarm was too loud at [their] house and $78 \%$ said that they felt they were not properly notified of the impact this windfarm would have on the community.....

Case 2, first person report by female, 2009

My home now sits among huge, massive turbines. Sixteen turbines surround me, all within a 3 $\mathrm{km}$ radius of my home. The closest is 400 metres from my back door. People often ask me what my problem is with the turbines. ("They are not very noisy," I am told.)

The noise is constant, some days louder than others. It is not noise I enjoy or choose to be around. It is noise I cannot escape. 
I live with the movement of shadow flicker created by the rotation of the turbines, coming through my dining room window as I drink my coffee in the morning. I have developed a sensitivity in which now I cannot even tolerate the movement of a small ceiling fan.

The skies where I live are no longer clear but dotted with blinking red lights marking the height of the turbines. When the turbines are down, a constant buzzing noise is emitted from the motionless structures. I have developed tinitus [sic] in my ears. I hear and feel the pulsating of the turbines and buzzing in my ears. I also feel the pulsating in my throat and chest.

Two homes have been abandoned where I live because of health reasons related to the effects of the turbines. One of these properties is host to 2 turbines. Many properties are for sale. In fact most of the properties where landowners reside on premises are for sale.

Real estate sales in my area are significantly less than other [similar places]. Some real estate brokers will not touch a property adjacent to a turbine for fear of future law suit. Nothing is selling in Turbine Town. Land value has decreased significantly because of the turbines.

I have:

- nausea (often) \& dizziness (often) 
- significant hearing loss

- itchy eyes

- high blood pressure (recently, an immediate and intense elevation to $180 / 118$, causing severe headache and complete dysfunction)

- heart palpitations

- achy joints

- short term memory loss

- severe sleep deprivation on a regular basis

Results of a sleep study I had done showed 214 interruptions in a 6 hour period (note: $6-8$ is considered normal; 214 is comparable to someone who has attention deficit disorder). I have very little if any regenerative sleep periods. I have been told that I have developed a sensitivity that does not leave my body when I leave the vicinity of the turbines. The term used was "toxic" -- my body is in a toxic state.

I have an ulcer in my nose that does not heal. I am awaiting an appointment in November with an ears, nose and throat specialist (otolaryngologist).

I often have blood in my urine (never was a problem in the past). I am having problems with my lymph nodes. I have been anaemic because of excessive blood loss. Blood work and other tests do not indicate changes which may cause this haemorrhaging. I have spent time in the emergency room at the hospital because of this. 
I once thought my degenerating health was part of the natural aging process. I did not believe the turbines could be the cause of my health issues. I questioned myself as to whether or not it was all in my head. I now believe exposure to the turbines accelerate these processes as well as create other health problems.

I am angry, helpless, and disappointed our government would let something like this happen. I am appalled at their ignorance and lack of compassion. It saddens me to watch my family and friends suffer from the same effects of the turbines.

It is also very saddening for me to see my dogs suffering. I cannot imagine the distress they must be enduring because of their sensitive hearing. I have not figured out what to do about it.

I spend as much time as I can away from my home, away from my son who is also sleep deprived and moody. We are exhausted and miserable. I often seek refuge with friends, often falling asleep minutes after I arrive. They are very understanding

\section{Case 3, first person report by male, 2010}

I am an abutter to ..., a $1.65 \mathrm{MW}$ Vestas 400 foot tall goliath. Since it went into operation in early 2010, quite a number of us abutters have suffered serious medical detriments and a gigantic loss of quality of our lives from the noise impact of this machine. 
My own home is 1662 feet from the turbine, and the effects of the sound on me have caused -anxiety

-stress

-nervousness

-sleep deprivation

-hypertension

-migraines

-dizziness

-blurred vision

-palpitations

-irritability

-anger

-upset stomach

-depression

These ailments are well documented by my medical providers.

The noise these turbines make is unlike regular noise. It is not the loudness of the noise but a characteristic to it that gets in your head and becomes entrenched. The sound can go on for days, 
or it can be absent, or it can be intermittent. When it is not there, one listens for it and is fearful of its return.

The garden that was a sanctuary to me for 30 years is now more like a torture chamber. Some of the abutters have started using the term "turbine torture." When the turbine first went into operation in March 2010, and then through April, I tried to acclimate myself to live with this thing.

After dropping into a three-month depression, I finally avoided my own home for the month of August, and pulled out of the depression. I returned on Labor Day weekend to find that after ten minutes of hearing the turbine, my anxiety and panic condition were returning. At least two persons have thought of suicide while this issue drags on through the creep of political process.

Anyone out there whose town or neighbor is proposing a wind turbine, I recommend for you to do your homework now before the machine is up and running, and you begin to plan to sell your home. I have been told, by the way, that if you are trying to sell and a turbine is visible from your home, your potential buyer list will drop by $50 \%$. 